\title{
Game Russian Options for Double Exponential Jump Diffusion Processes
}

\author{
Atsuo Suzuki ${ }^{*}$, Katsushige Sawaki ${ }^{2}$ \\ ${ }^{1}$ Meijo University, Gifu, Japan \\ ${ }^{2}$ Aoyama Gakuin University, Sagamihara, Japan \\ Email: ${ }^{*}$ atsuo@urban.meijo-u.ac.jp, sawaki@si.aoyama.ac.jp
}

Received November 8, 2013; revised December 11, 2013; accepted December 27, 2013

Copyright (C) 2014 Atsuo Suzuki, Katsushige Sawaki. This is an open access article distributed under the Creative Commons Attribution License, which permits unrestricted use, distribution, and reproduction in any medium, provided the original work is properly cited. In accordance of the Creative Commons Attribution License all Copyrights (C) 2014 are reserved for SCIRP and the owner of the intellectual property Atsuo Suzuki, Katsushige Sawaki. All Copyright (C) 2014 are guarded by law and by SCIRP as a guardian.

\section{ABSTRACT}

In this paper, we deal with the valuation of Game Russian option with jumps, which is a contract that the seller and the buyer have both the rights to cancel and to exercise it at any time, respectively. This model can be formulated as a coupled optimal stopping problem. First, we discuss the pricing model with jumps when the stock pays dividends continuously. Secondly, we derive the value function of Game Russian options and investigate properties of optimal boundaries of the buyer. Finally, some numerical results are presented to demonstrate analytical properties of the value function.

\section{KEYWORDS}

\section{Stochastic Process; Game Russian Option; Double Exponential Distribution; Optimal Stopping; Optimal} Boundaries

\section{Introduction}

Russian option was introduced by Shepp and Shiryaev [1,2] and it was one of perpetual American lookback options. In Russian option, the buyer has the right to exercise it at any time. On the other hand, in Game Russian option, not only the buyer but also the seller has the right to cancel it at any time. This option is based on Game option introduced by Kifer [3]. Game option frame work can be applied to various American-type options. Therefore, we apply this frame work to Russian option. The valuation of Game Russian option can be formulated as a coupled optimal stopping problem. See Cvitanic and Karatzas [4], Kifer [3].

Kyprianou [5] derived the closed-form solution in the case where the dividend rate is zero. Suzuki and Sawaki [6] gave the pricing formula with positive dividend. Kou and Wang [7] presented the closed-form for the value function of perpetual American put options without dividend and so on. Suzuki and Sawaki [8] studied the pricing formula of Russian option for double exponential jump diffusion processes.

In this paper, we deal with Game Russian options. Game Russian option is a contact that the seller and the buyer have the rights to cancel and to exercise it at any time, respectively. We present the pricing formula of Game Russian options for double exponential jump diffusion processes. The pricing of such an option can be formulated as a coupled optimal stopping problem which is analyzed as Dynkin game. We derive the value function of Game Russian option and its optimal boundaries. Also some numerical results are presented to demonstrate analytical sensitivities of the value function with respect to parameters.

This paper is organized as follows. In Section 2, we introduce a pricing model of Game Russian options by means of a coupled optimal stopping problem given by Kifer [3]. Section 3 presents the value function of Game Russian options for double exponential jump diffusion processes. Section 4 presents numerical examples to ver-

\footnotetext{
${ }^{*}$ Corresponding author.
} 
ify analytical results. We end the paper with some concluding remarks and future work.

\section{Pricing Model}

In this section, we consider the pricing model for Game Russian option. Let $B(t)$ be the process of the riskless asset price at time $t$ defined by $B(t)=B(0) \mathrm{e}^{r t}$, where $r$ is the positive interest rate. Let $W(t)$ be a standard Brownian motion and $N(t)$ be a Poisson process with the intensity $\lambda$. Let $J_{i}$ denote i.i.d. positive random variables. $Y_{i} \equiv \log J_{i}$ has a double exponential distribution and its density function is given by

$$
f(y)=p \eta_{1} \mathrm{e}^{-\eta_{1} y} 1_{\{y \geq 0\}}+q \eta_{2} \mathrm{e}^{\eta_{2} y} 1_{\{y<0\}},
$$

where $\eta_{1}>1, \eta_{2}>0$ and $0 \leq p, q \leq 1$ such that $p+q=1$. Under a risk-neutral probability, the process of the risky asset price $S(t)$ at time $t$ satisfies the stochastic differential equation

$$
\frac{\mathrm{d} S(t)}{S(t-)}=\mu \mathrm{d} t+\kappa \mathrm{d} W(t)+\mathrm{d}\left(\sum_{i=1}^{N(t)}\left(J_{i}-1\right)\right),
$$

where $\mu$ and $\kappa>0$ are constants. Define another probability measure $\tilde{P}$ as

$$
\left.\frac{\mathrm{d} \tilde{P}}{\mathrm{~d} P}\right|_{\mathcal{F}_{t}}=\exp \left\{-b W(t)-\frac{1}{2} b^{2} t\right\}, b=\frac{\mu-r+d+\lambda \zeta}{\kappa},
$$

where $d(<r)$ is a nonnegative continuous dividend rate of the risky asset, $\mathcal{F}(t)=\sigma\left(W(s), N(s), s \leq t,\left\{J_{i}\right\}\right)$ is the information available at time $t$ and

$$
\zeta=E\left[J_{i}\right]-1=\frac{p \eta_{1}}{\eta_{1}-1}+\frac{q \eta_{2}}{\eta_{2}+1}-1 .
$$

By Girsanov's theorem, $\tilde{W}(t)=W(t)-b t$ is a Brownian motion with respect to $\tilde{P}$.

We can rewrite (1) as

$$
\frac{\mathrm{d} S(t)}{S(t-)}=(r-d-\lambda \zeta) \mathrm{d} t+\kappa \mathrm{d} \tilde{W}(t)+d\left(\sum_{i=1}^{N(t)}\left(J_{i}-1\right)\right) .
$$

Solving (2) gives $S(t)=S(0) \exp X(t)$, where

$$
X(t)=\left(r-d-\frac{1}{2} \kappa^{2}-\lambda \zeta\right) t+\kappa \tilde{W}(t)+\sum_{i=1}^{N(t)} Y_{i}
$$

Let $V(v)$ be a function of class $C^{2}$. Then the infinitesimal generator $\mathcal{L}$ of the process $S(t)$ is given by

$$
\mathcal{L} V(v)=\frac{1}{2} \kappa^{2} v^{2} V^{\prime \prime}(v)+(r-d-\lambda \zeta) v V^{\prime}(v)+\lambda \int_{-\infty}^{\infty}\left(V\left(v \mathrm{e}^{y}\right)-V(v)\right) f(y) \mathrm{d} y
$$

for all $v>0$.

Next we introduce the four real numbers $\beta_{1}, \beta_{2}, \beta_{3}, \beta_{4}$. Kou and Wang [9] showed that the equation $G(\theta)=\alpha$ for all $\alpha>0$ has the solutions $\beta_{1}, \beta_{2},-\beta_{3},-\beta_{4}$, where

$$
G(\theta)=\theta\left(r-d-\frac{1}{2} \kappa^{2}-\lambda \zeta\right)+\frac{1}{2} \theta^{2} \kappa^{2}+\lambda\left(\frac{p \eta_{1}}{\eta_{1}-\theta}+\frac{q \eta_{2}}{\eta_{2}+\theta}-1\right) .
$$

And the four solutions satisfy the following inequalities

$$
0<\beta_{1}<\eta_{1}<\beta_{2}<\infty, 0<\beta_{3}<\eta_{2}<\beta_{4}<\infty .
$$

Remark 2.1 When the dividend rate $d=0, \beta_{1}=1$.

Define the process

$$
\Psi(t) \equiv \max \left(v s, \sup _{0 \leq u \leq t} S(u)\right) / S(t), S(0)=s, v \geq 1 .
$$

Then the value function of Russian option is given by

$$
V_{R}(v)=\sup _{\tau} \tilde{E}\left[\mathrm{e}^{-\alpha \tau} \Psi(\tau) \mid \Psi(0)=v\right],
$$


where the supremum is taken for all stopping times $\tau$.

Theorem 2.1 (Suzuki and Sawaki [8]) The value function $V_{R}(v)$ of Russian option with jump is given by $V_{R}(v)= \begin{cases}A\left(v_{1}\right) v^{\beta_{1}}+B\left(v_{1}\right) v^{\beta_{2}}+C\left(v_{1}\right) v^{-\beta_{3}}+D\left(v_{1}\right) v^{-\beta_{4}}, & 1 \leq v \leq v_{1} \\ v, & v \geq v_{1} .\end{cases}$

The coefficients are determined by

$$
\begin{aligned}
& A\left(v_{1}\right)=\frac{\left(\eta_{1}-\beta_{1}\right) v_{1}^{-\beta_{1}}}{\left(\beta_{1}+\beta_{3}\right)\left(\beta_{2}-\beta_{1}\right)}\left\{\frac{\left(\beta_{2}-1\right)\left(\beta_{3}+1\right)}{\eta_{1}-1} v_{1}-\frac{\left(\beta_{2}+\beta_{4}\right)\left(\beta_{4}-\beta_{3}\right)}{\eta_{1}+\beta_{4}} D v_{1}^{-\beta_{4}}\right\} \\
& B\left(v_{1}\right)=\frac{\left(\beta_{2}-\eta_{1}\right) v_{1}^{-\beta_{2}}}{\left(\beta_{2}-\beta_{1}\right)\left(\beta_{2}+\beta_{3}\right)}\left\{\frac{\left(\beta_{1}-1\right)\left(\beta_{3}+1\right)}{\eta_{1}-1} v_{1}-\frac{\left(\beta_{1}+\beta_{4}\right)\left(\beta_{4}-\beta_{3}\right)}{\eta_{1}+\beta_{4}} D v_{1}^{-\beta_{4}}\right\} \\
& C\left(v_{1}\right)=\frac{\left(\eta_{1}+\beta_{3}\right) v_{1}^{\beta_{3}}}{\left(\beta_{1}+\beta_{3}\right)\left(\beta_{2}+\beta_{3}\right)}\left\{\frac{\left(\beta_{1}-1\right)\left(\beta_{2}-1\right)}{\eta_{1}-1} v_{1}-\frac{\left(\beta_{1}+\beta_{4}\right)\left(\beta_{2}+\beta_{4}\right)}{\eta_{1}+\beta_{4}} D v_{1}^{-\beta_{4}}\right\}
\end{aligned}
$$

and

$$
\frac{A\left(v_{1}\right)}{\eta_{2}+\beta_{1}}+\frac{B\left(v_{1}\right)}{\eta_{2}+\beta_{2}}+\frac{C\left(v_{1}\right)}{\eta_{2}-\beta_{3}}+\frac{D\left(v_{1}\right)}{\eta_{2}-\beta_{4}}=0
$$

Moreover, the optimal boundary $v_{1}$ is the solution in $(1, \infty)$ to the equation

$$
A(v) \beta_{1}+B(v) \beta_{2}-C(v) \beta_{3}-D(v) \beta_{4}=0
$$

and the optimal stopping time is given by

$$
\tilde{\tau}=\inf \left\{t>0 \mid \Psi(t) \geq v_{1}\right\}
$$

\section{Game Russian Options}

Let $\sigma$ denote a cancel time for the seller and $\tau$ an exercise time for the buyer. If the seller cancels the contract, the buyer receives $\Psi(\sigma)+\delta$ from the seller. We can think of $\delta>0$ as the penalty cost for the cancellation. On the other hand, if the buyer exercises it, (s)he receives $\Psi(\tau)$ from the seller. Therefore, the payoff function for the buyer is given by

$$
(\Psi(\sigma)+\delta) 1_{\{\sigma<\tau\}}+\Psi(\tau) 1_{\{\tau \leq \sigma\}} .
$$

Let $\mathcal{T}_{0, \infty}$ denote the set of all stopping times with values in the interval $[0, \infty]$. Then the value function $V^{*}(v)$ of Game Russian option is defined by

$$
V^{*}(v)=\inf _{\sigma \in \mathcal{T}_{0, \infty}} \sup _{\tau \in \mathcal{T}_{0, \infty}} J(\sigma, \tau, v),
$$

where

$$
J(\sigma, \tau, v)=\tilde{E}\left[\mathrm{e}^{-\alpha(\sigma \wedge \tau)}\left\{(\Psi(\sigma)+\delta) 1_{\{\sigma<\tau\}}+\Psi(\tau) 1_{\{\tau \leq \sigma\}}\right\} \mid \Psi(0)=v\right], \alpha>0 .
$$

And the function $V^{*}(v)$ satisfies the inequalities

$$
v \leq V^{*}(v) \leq v+\delta,
$$

which provides the lower and the upper bounds for the value function of Game Russian option.

We define two sets $A$ and $B$ as

$$
\begin{gathered}
A=\left\{v \in \mathbf{R}^{+} \mid V^{*}(v)=v+\delta\right\} \\
B=\left\{v \in \mathbf{R}^{+} \mid V^{*}(v)=v\right\} .
\end{gathered}
$$

$A$ and $B$ are called the seller's cancellation region and the buyer's exercise region, respectively. Then the two optimal stopping times are given by

$$
\sigma_{A}=\inf \{t>0 \mid \Psi(t) \in A\},
$$




$$
\tau_{B}=\inf \{t>0 \mid \Psi(t) \in B\} \text {. }
$$

Then for any $v, \hat{\sigma} \equiv \sigma_{A}$ and $\hat{\tau} \equiv \tau_{B}$ attain the infimum and supremum in (3), i.e., we have $V^{*}(v)=J(\hat{\sigma}, \hat{\tau}, v)$.

The pair $(\hat{\sigma}, \hat{\tau})$ is the saddle point of $J(\sigma, \tau, v)$.

Remark 3.1 The seller minimizes the payoff function and $\Psi(t) \geq \Psi(0)=v \geq 1$. From this, it follows that the seller's optimal cancellation region is $\{1\}$.

Lemma 3.1 Suppose that $r-d-\frac{1}{2} \kappa^{2}-\lambda \zeta>0$. Then the function $V^{*}(v)$ is Lipschitz continuous and its Radon-Nikodym derivative satisfies

$$
0 \leq \frac{\mathrm{d} V^{*}(v)}{\mathrm{d} v} \leq 1, \text { a.e.v. }
$$

Proof. Since $\hat{\sigma}, \hat{\tau}$ and $\Psi(t)$ depend on the initial value $v$, we write them as $\hat{\sigma}^{v}, \hat{\tau}^{v}$ and $\Psi(t, v)$. Replacing the optimal stopping times $\hat{\tau}^{v}$ by another stopping time $\hat{\tau}^{u}$, we get the inequalities $V^{*}(v) \leq J\left(\hat{\sigma}^{u}, \hat{\tau}^{v}, v\right), V^{*}(u) \geq J\left(\hat{\sigma}^{u}, \hat{\tau}^{v}, u\right)$.

Note that $z_{1}^{+}-z_{2}^{+} \leq\left(z_{1}-z_{2}\right)^{+}$for any $z_{1}, z_{2} \in \mathbf{R}$. For any $v>u$, we have

$$
\begin{aligned}
0 & \leq V^{*}(v)-V^{*}(u) \\
& =J\left(\hat{\sigma}^{u}, \hat{\tau}^{v}, v\right)-J\left(\hat{\sigma}^{u}, \hat{\tau}^{v}, u\right) \\
& =\tilde{E}\left[\mathrm{e}^{-\alpha\left(\hat{\sigma}^{u} \wedge \hat{\tau}^{v}\right)}\left(\Psi\left(\hat{\sigma}^{u} \wedge \hat{\tau}^{v}, v\right)-\Psi\left(\hat{\sigma}^{u} \wedge \hat{\tau}^{v}, u\right)\right)\right] \\
& =\tilde{E}\left[\mathrm{e}^{-\alpha\left(\hat{\sigma}^{u} \wedge \hat{\tau}^{v}\right)} H^{-1}\left(\hat{\sigma}^{u} \wedge \hat{\tau}^{v}\right)\left\{\left(v-\sup H_{u}\right)^{+}-\left(u-\sup H_{u}\right)^{+}\right\}\right] \\
& \leq(v-u) \tilde{E}\left[\mathrm{e}^{-\alpha\left(\hat{\sigma}^{u} \wedge \hat{\tau}^{v}\right)} H^{-1}\left(\hat{\sigma}^{u} \wedge \hat{\tau}^{v}\right)\right] \\
& \leq v-u,
\end{aligned}
$$

where $H(t)=\exp X(t)$. Therefore, we obtain

$$
0 \leq \frac{V^{*}(v)-V^{*}(u)}{v-u} \leq 1 .
$$

This means that $V^{*}(v)$ is Lipschitz continuous and satisfies (4).

If the penalty $\delta$ is large enough, the seller never cancels. It is of interest to show how much delta should be large for the seller never to cancel.

Lemma 3.2 Set $\delta^{*}=V_{R}(1)-1$. If the penalty $\delta>\delta^{*}$, the seller never cancels. In other words, Game Russian option is reduced to Russian option.

Proof. Consider the function $U(v)=V_{R}(v)-v-\delta$. Since it holds $U^{\prime}(v) \leq 0$ by Lemma 3.1 and $U(1)=\delta^{*}-\delta<0$, we have $V_{R}(v)<v+\delta$. Hence, it follows that $V^{*}(v)<v+\delta$ because the inequality $V^{*}(v) \leq V_{R}(v)$ holds.

We assume that $p=1$ and $q=0$. It means that the jump occurs only upward. This is very useful to analyse stochastic cash management problem for jump diffusion processes (See Sato and Suzuki [10]). Then we can express $G(\theta)$ as

$$
G(\theta)=\theta\left(r-d-\frac{1}{2} \kappa^{2}-\lambda \zeta\right)+\frac{1}{2} \theta^{2} \kappa^{2}+\lambda\left(\frac{\eta_{1}}{\eta_{1}-\theta}-1\right)
$$

and the equation $G(\theta)=\alpha$ has three solutions $\beta_{1}, \beta_{2},-\beta_{4}$, which satisfy

$$
1 \leq \beta_{1}<\eta_{1}<\beta_{2}<\infty, 0<\beta_{4}<\infty .
$$

We introduce the function for $v_{0}=\mathrm{e}^{x_{0}}>1$

$$
V(v)= \begin{cases}A v^{\beta_{1}}+B v^{\beta_{2}}+C v^{-\beta_{4}}, & 1 \leq v \leq v_{0} \\ v, & v \geq v_{0}\end{cases}
$$


We set $v=\mathrm{e}^{x}$ and $V(v)=V\left(\mathrm{e}^{x}\right) \equiv \hat{V}(x)$. In what follows, we determine the coefficients $A, B, C$ and $\mathrm{e}^{x_{0}}$. In order to determine the coefficients, we prepare the conditions. By value matching condition, we have

$$
A \mathrm{e}^{\beta_{1} x_{0}}+B \mathrm{e}^{\beta_{2} x_{0}}+C \mathrm{e}^{-\beta_{4} x_{0}}=\mathrm{e}^{x_{0}}
$$

and by smooth pasting condition, we have

$$
A \beta_{1} \mathrm{e}^{\beta_{1} x_{0}}+B \beta_{2} \mathrm{e}^{\beta_{2} x_{0}}-C \beta_{4} \mathrm{e}^{-\beta_{4} x_{0}}=\mathrm{e}^{x_{0}} .
$$

We can get the last condition by using the infinitesimal generator $L$ of the process $X(t)$ given by

$$
\hat{\mathcal{L}} \hat{V}(x)=\frac{1}{2} \kappa^{2} \hat{V}^{\prime \prime}(x)+\left(r-d-\frac{1}{2} \kappa^{2}-\lambda \zeta\right) \hat{V}^{\prime}(x)+\lambda \int_{-\infty}^{\infty}(\hat{V}(x+y)-\hat{V}(x)) f(y) \mathrm{d} y
$$

for all $x>0$. For $x<x_{0}$, we obtain

$$
\begin{aligned}
\int_{-\infty}^{\infty} \hat{V}(x+y) f(y) \mathrm{d} y \\
=\int_{0}^{x_{0}-x}\left(A \mathrm{e}^{\beta_{1}(x+y)}+B \mathrm{e}^{\beta_{2}(x+y)}+C \mathrm{e}^{-\beta_{4}(x+y)}\right) \eta_{1} \mathrm{e}^{-\eta_{1} y} \mathrm{~d} y+\int_{x_{0}-x}^{\infty} \mathrm{e}^{x+y} \eta_{1} \mathrm{e}^{-\eta_{1} y} \mathrm{~d} y \\
=\eta_{1}\left(\frac{A}{\eta_{1}-\beta_{1}} \mathrm{e}^{\beta_{1} x}+\frac{B}{\eta_{1}-\beta_{2}} \mathrm{e}^{\beta_{2} x}+\frac{C}{\eta_{1}+\beta_{4}} \mathrm{e}^{-\beta_{4} x}\right) \\
\\
-\eta_{1} \mathrm{e}^{-\eta_{1}\left(x_{0}-x\right)}\left(\frac{A}{\eta_{1}-\beta_{1}} \mathrm{e}^{\beta_{1} x_{0}}+\frac{B}{\eta_{1}-\beta_{2}} \mathrm{e}^{\beta_{2} x_{0}}+\frac{C}{\eta_{1}+\beta_{4}} \mathrm{e}^{-\beta_{4} x_{0}}-\frac{\mathrm{e}^{x_{0}}}{\eta_{1}-1}\right) .
\end{aligned}
$$

From this, we obtain

$$
\begin{aligned}
& (\hat{L}-r) \hat{V}(x) \\
= & A \mathrm{e}^{\beta_{1} x}\left(\frac{1}{2} \beta_{1}^{2}+\beta_{1}\left(r-d-\frac{1}{2} \kappa^{2}-\lambda \zeta\right)\right)+B \mathrm{e}^{\beta_{2} x}\left(\frac{1}{2} \beta_{2}^{2}+\beta_{2}\left(r-d-\frac{1}{2} \kappa^{2}-\lambda \zeta\right)\right) \\
& +C \mathrm{e}^{-\beta_{4} x}\left(\frac{1}{2}\left(-\beta_{4}\right)^{2}-\beta_{4}\left(r-d-\frac{1}{2} \kappa^{2}-\lambda \zeta\right)\right)+\lambda \int_{-\infty}^{\infty} \hat{V}(x+y) f(y) \mathrm{d} y-(\lambda+r) \hat{V}(x) \\
= & A \mathrm{e}^{\beta_{1} x} g\left(\beta_{1}\right)+B \mathrm{e}^{\beta_{2} x} g\left(\beta_{2}\right)+C \mathrm{e}^{-\beta_{4} x} g\left(-\beta_{4}\right) \\
& -\lambda p \eta_{1} \mathrm{e}^{-\eta_{1}\left(x_{0}-x\right)}\left(\frac{A}{\eta_{1}-\beta_{1}} \mathrm{e}^{\beta_{1} x_{0}}+\frac{B}{\eta_{1}-\beta_{2}} \mathrm{e}^{\beta_{2} x_{0}}+\frac{C}{\eta_{1}+\beta_{4}} \mathrm{e}^{-\beta_{4} x_{0}}-\frac{\mathrm{e}^{x_{0}}}{\eta_{1}-1}\right),
\end{aligned}
$$

where $g(x)=G(-x)-r$. By Lemma 2.1 in Kou and Wang [9], we have $g\left(\beta_{1}\right)=g\left(\beta_{2}\right)=g\left(-\beta_{4}\right)=0$. Since $(L-r) \hat{V}(x)=0$ holds, we get the condition

$$
\frac{A}{\eta_{1}-\beta_{1}} \mathrm{e}^{\beta_{1} x_{0}}+\frac{B}{\eta_{1}-\beta_{2}} \mathrm{e}^{\beta_{2} x_{0}}+\frac{C}{\eta_{1}+\beta_{4}} \mathrm{e}^{-\beta_{4} x_{0}}-\frac{\mathrm{e}^{x_{0}}}{\eta_{1}-1}=0 .
$$

Lemma 3.3 Solving the following equations

$$
\begin{aligned}
& A \mathrm{e}^{\beta_{1} x_{0}}+B \mathrm{e}^{\beta_{2} x_{0}}+C \mathrm{e}^{-\beta_{4} x_{0}}=\mathrm{e}^{x_{0}} \\
& A \beta_{1} \mathrm{e}^{\beta_{1} x_{0}}+B \beta_{2} \mathrm{e}^{\beta_{2} x_{0}}-C \beta_{4} \mathrm{e}^{-\beta_{4} x_{0}}=\mathrm{e}^{x_{0}} \\
& \frac{A}{\eta_{1}-\beta_{1}} \mathrm{e}^{\beta_{1} x_{0}}+\frac{B}{\eta_{1}-\beta_{2}} \mathrm{e}^{\beta_{2} x_{0}}+\frac{C}{\eta_{1}+\beta_{4}} \mathrm{e}^{-\beta_{4} x_{0}}=\frac{\mathrm{e}^{x_{0}}}{\eta_{1}-1}
\end{aligned}
$$

gives the solutions

$$
\begin{aligned}
& A=\frac{\left(\eta_{1}-\beta_{1}\right)\left(\beta_{2}-1\right)\left(\beta_{4}+1\right)}{\left(\eta_{1}-1\right)\left(\beta_{1}+\beta_{4}\right)\left(\beta_{2}-\beta_{1}\right)} \mathrm{e}^{\left(1-\beta_{1}\right) x_{0}} \\
& B=\frac{\left(\beta_{2}-\eta_{1}\right)\left(\beta_{1}-1\right)\left(\beta_{4}+1\right)}{\left(\eta_{1}-1\right)\left(\beta_{2}+\beta_{4}\right)\left(\beta_{2}-\beta_{1}\right)} \mathrm{e}^{\left(1-\beta_{2}\right) x_{0}} \\
& C=\frac{\left(\eta_{1}+\beta_{4}\right)\left(\beta_{2}-1\right)\left(\beta_{1}-1\right)}{\left(\eta_{1}-1\right)\left(\beta_{1}+\beta_{4}\right)\left(\beta_{2}+\beta_{4}\right)} \mathrm{e}^{\left(1+\beta_{4}\right) x_{0}} .
\end{aligned}
$$


Since the coefficients $A, B, C$ depend on $x_{0}$, we denote them as $A\left(x_{0}\right), B\left(x_{0}\right)$ and $C\left(x_{0}\right)$. The number $v_{0}=\mathrm{e}^{x_{0}}$ given by (5) satisfies the equation

$$
A\left(x_{0}\right) \mathrm{e}^{\beta_{1} x_{0}}+B\left(x_{0}\right) \mathrm{e}^{\beta_{2} x_{0}}+C\left(x_{0}\right) \mathrm{e}^{-\beta_{4} x_{0}}=\delta+1 .
$$

\section{Main Theorem}

Theorem 4.1 Let $V^{*}(v)$ denote the value function of Game Russian option. If $\delta \geq \delta^{*}$, the value function is equal to the one of Russian option, i.e. $V^{*}(v)=V_{R}(v)$. If $\delta<\delta^{*}$, then $V^{*}(v)$ is given by

$$
V^{*}(v)= \begin{cases}A\left(v_{0}\right) v^{\beta_{1}}+B\left(v_{0}\right) v^{\beta_{2}}+C\left(v_{0}\right) v^{-\beta_{4}}, & 1 \leq v \leq v_{0} \\ v, & v \geq v_{0}\end{cases}
$$

and the optimal stopping times are given by

$$
\begin{aligned}
& \hat{\sigma}=\inf \{t>0 \mid \Psi(t)=1\}, \\
& \hat{\tau}=\inf \left\{t>0 \mid \Psi(t) \geq v_{0}\right\} .
\end{aligned}
$$

The optimal boundary $v_{0}$ for the buyer is the unique solution to the equation

$$
A(v)+B(v)+C(v)=\delta+1 .
$$

In order to prove the above theorem, we need the following lemmas.

Lemma 4.1 Assume that a function $V(v)$ has the following properties;

1) $V(v)=v$ and $(\mathcal{L}-r) V(v) \leq 0$, for $v>v_{0}$.

2) It holds $(\mathcal{L}-r) V(v)=0$ and $V(v)$ satisfies $v<V(v)<v+\delta$ for $1<v<v_{0}$.

3) At $v=v_{0}$ we have $V^{\prime}\left(v_{0}-\right)=V^{\prime}\left(v_{0}+\right)$.

Then, $V$ is the value function of Game Russian options with dividend, i.e., $V^{*}=V$ holds. The optimal exercise region is the interval $\left[v_{0}, \infty\right)$ and the optimal cancellation region is $\{1\}$.

Proof. By Ito's formula, we have

$$
\mathrm{e}^{-\alpha t} V(\Psi(t))-V(\Psi(t))=\text { martingale with } 0+\int_{0}^{t} \mathrm{e}^{-\alpha u}(\mathcal{L}-r) V(\Psi(u)) \mathrm{d} u .
$$

Set

$$
\hat{\sigma}=\inf \{t>0 ; \Psi(t)=1\}, \hat{\tau}=\inf \left\{t>0 ; \Psi(t) \in\left[v_{0}, \infty\right]\right\}
$$

and $t=\hat{\sigma} \wedge \hat{\tau}$. Since $(\mathcal{L}-r) V(\Psi(u))=0$ a.s. for $u<t$, we have $\int_{0}^{t} \mathrm{e}^{-\alpha u}(\mathcal{L}-r) V(\Psi(u)) \mathrm{d} u=0$ a.s. Therefore, taking expectation of (8), we have

$$
V(v)=\tilde{E}\left[\mathrm{e}^{-\alpha(\hat{\sigma} \wedge \hat{\tau})} V(\Psi(\hat{\sigma} \wedge \hat{\tau})) \mid \Psi(0)=v\right] .
$$

It holds

$$
V(\Psi(\hat{\sigma} \wedge \hat{\tau}))=\Psi(\hat{\tau}) 1_{\{\hat{\imath} \leq \hat{\sigma}\}}+(\Psi(\hat{\sigma})+\delta) 1_{\{\hat{\sigma}<\hat{\tau}\}} .
$$

Therefore we get $V(v)=J(\hat{\sigma}, \hat{\tau}, v)$.

For any $\tau$, set $t=\hat{\sigma} \wedge \tau$. The term of $\mathrm{d} u$ is nonpositive a.s. because $(\mathcal{L}-r) V(v) \leq 0$. Taking expectation of (8), we get

$$
\tilde{E}\left[\mathrm{e}^{-\alpha(\hat{\sigma} \wedge \tau)} V(\Psi(\hat{\sigma} \wedge \tau))\right] \leq V(v) .
$$

The above left hand side dominates $J(\hat{\sigma}, \tau, v)$. Therefore

$$
\inf _{\sigma} \sup _{\tau} J(\sigma, \tau, v) \leq \sup _{\tau} J(\hat{\sigma}, \tau, v) \leq V(v) .
$$

Next for any $\sigma$, set $t=\sigma \wedge \hat{\tau}$. Similarly it holds

$$
\tilde{E}\left[\mathrm{e}^{-\alpha(\sigma \wedge \hat{\tau})} V(\Psi(\sigma \wedge \hat{\tau}))\right] \geq V(v) .
$$

Since the left hand side is dominated by $J(\sigma, \hat{\tau}, v)$, we get 


$$
\inf _{\sigma} \sup _{\tau} J(\sigma, \tau, v) \geq \inf _{\sigma} J(\sigma, \hat{\tau}, v) \geq V(v) .
$$

From (9) and (10), we have $\inf _{\sigma} \sup _{\tau} J(\sigma, \tau, v)=V(v)$.

Lemma 4.2 The function $V(v)$ satisfies $(\mathcal{L}-r) V(v) \leq 0$

Proof. Since $\hat{V}(x)=\mathrm{e}^{x}$ for $x>x_{0}$, we have

$$
\int_{0}^{\infty} \hat{V}(x+y) f(y) \mathrm{d} y=\int_{0}^{\infty} \eta_{1} \mathrm{e}^{x+\left(1-\eta_{1}\right) y} \mathrm{~d} y=\frac{\eta_{1} \mathrm{e}^{x}}{\eta_{1}-1} .
$$

Hence, we obtain

$$
(\hat{\mathcal{L}}-r) \hat{V}(x)=\frac{1}{2} \kappa^{2} \mathrm{e}^{x}+\left(r-d-\frac{1}{2} \kappa^{2}-\lambda \zeta\right) \mathrm{e}^{x}+\frac{\lambda \eta_{1}}{\eta_{1}-1} \mathrm{e}^{x}-(\lambda+r) \mathrm{e}^{x}=-\mathrm{de}^{x} \leq 0 .
$$

That is, we obtain $(\mathcal{L}-r) V(v) \leq 0$.

Lemma 4.3 For $1<v<v_{0}$ the function $V(v)$ satisfies $(\mathcal{L}-r) V(v)=0$ and $v<V(v)<v+\delta$.

Proof. The former assertion is known. We shall show the latter one. The second derivative of $V(v)$ is nonnegative because $\beta_{1}, \beta_{2}>1$ and $A, B, C>0$. It follows that $V$ is a convex function. Since $V(v)$ is a convex function, $V^{\prime}(v)$ is increasing. From this, we can see that $V^{\prime}(v)<1$ for $1<v<v_{0}$. By the boundary conditions $V(1)=\delta+1$ and $V\left(v_{0}\right)=v_{0}$, we have $v<V(v)<v+\delta$.

Lemma 4.4 Set

$$
h(v)=\delta+1-A(v)-B(v)-C(v) .
$$

Then the equation $h(v)=0$ has the unique solution in the interval $(1, \infty)$.

Proof. By (11), a direct computation yields

$$
\begin{aligned}
h(1) & =\delta+1-\frac{\left(\eta_{1}-\beta_{1}\right)\left(\beta_{2}-1\right)\left(\beta_{4}+1\right)}{\left(\eta_{1}-1\right)\left(\beta_{1}+\beta_{4}\right)\left(\beta_{2}-\beta_{1}\right)}-\frac{\left(\beta_{2}-\eta_{1}\right)\left(\beta_{1}-1\right)\left(\beta_{4}+1\right)}{\left(\eta_{1}-1\right)\left(\beta_{2}+\beta_{4}\right)\left(\beta_{2}-\beta_{1}\right)}-\frac{\left(\eta_{1}+\beta_{4}\right)\left(\beta_{2}-1\right)\left(\beta_{1}-1\right)}{\left(\eta_{1}-1\right)\left(\beta_{1}+\beta_{4}\right)\left(\beta_{2}+\beta_{4}\right)} \\
& =\delta>0 .
\end{aligned}
$$

Since $h^{\prime \prime}(v)<0$ and $h^{\prime}(1)=0$, we have $h^{\prime}(v)<0$. Furthermore, it holds $\lim _{v \rightarrow \infty} h(v)=-\infty$. Therefore, the equation $h(v)=0$ has the unique solution in $(1, \infty)$.

In the rest of this section, we present some numerical examples to demonstrate theoretical results and some effects of parameters on the price of Game Russian option. We set $r=0.1, d=0.09, \kappa=0.3, p=1, q=0$, $\eta_{1}=50, \lambda=3$. Using these parameters, $\delta^{*}$ is 0.248 .

Figure 1 shows how the optimal exercise boundary increase as the penalty $\delta$ increases from 0.1 up to $\delta^{*}$. From the figure, we can see that the optimal boundary $v_{0}$ is increasing in the penalty $\delta$. Figure 2 demonstra-

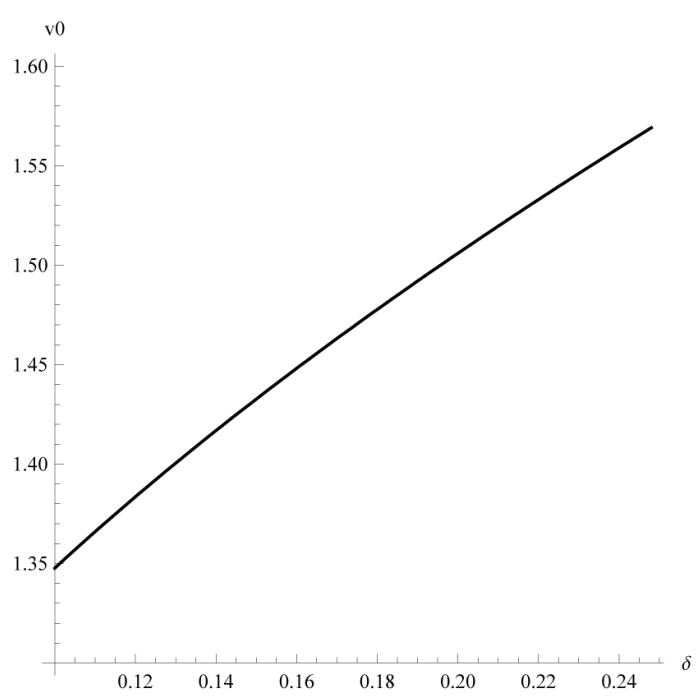

Figure 1. Optimal boundary for the buyer. 


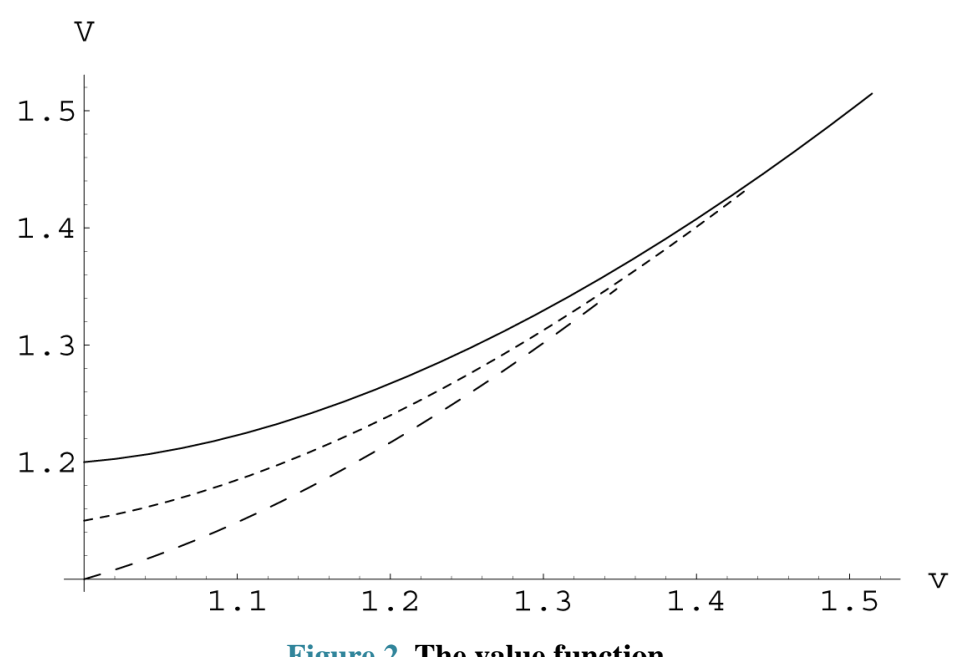

tes the value function of Game Russian option with jumps. Dashed lines represent the graph of the value $V$ in $\delta=0.1,0.15$ from the bottom, respectively. Real line represents the value in $\delta=0.2$. From Figure 2, we can visually recognize that $V^{*}(v)$ is convex and increasing in $v$.

\section{Conclusion}

In this paper, we discussed the valuation of Game Russian option written on dividend paying asset, obtained the value function of it for double exponential jump diffusion processes and also explored some analytical properties of the value function and the optimal boundaries for the seller and buyer, which were useful to provide an approximation of the finite lived Game Russian option. Moreover, we plan to examine convertible bonds with jumps by using Game option frame work. We shall leave it as future work.

\section{REFERENCES}

[1] Shepp, L.A. and Shiryaev, A.N. (1993) The Russian option: Reduced regret. The Annals of Applied Probability, 3, $631-640$. http://dx.doi.org/10.1214/aoap/1177005355

[2] Shepp, L.A. and Shiryaev, A.N. (1994) A new look at pricing of the "Russian option”. Theory of Probability and Its Applications, 39, 103-119. http://dx.doi.org/10.1137/1139004

[3] Kifer, Y. (2000) Game options. Finance and Stochastics, 4, 443-463. http://dx.doi.org/10.1007/PL00013527

[4] Cvitanic, J. and Karatzas, I. (1996) Backward stochastic differential equations with reflection and Dynkin games. The Annals of Probability, 24, 2024-2056. http://dx.doi.org/10.1214/aop/1041903216

[5] Kyprianou, A.E. (2004) Some calculations for Israeli options. Finance and Stochastics, 8, 73-86. http://dx.doi.org/10.1007/s00780-003-0104-5

[6] Suzuki, A. and Sawaki, K. (2009) The pricing of callable Russian options and their optimal boundaries. Journal of Applied Mathematics and Decision Sciences, 2009, Article ID: 593986, 13 p. http://dx.doi.org/10.1155/2009/593986

[7] Kou, S.G. and Wang, H. (2004) Option pricing under a double exponential jump diffusion model. Management Science, 50, 1178-1192. http://dx.doi.org/10.1287/mnsc.1030.0163

[8] Suzuki, A. and Sawaki, K. (2010) The valuation of Russian options for double exponential jump diffusion processes. Asia Pacific Journal of Operational Research, 27, 227-242.

[9] Kou, S.G. and Wang, H. (2003) First passage times for a jump diffusion process. Advances in Applied Probability, 35, $504-531$. http://dx.doi.org/10.1239/aap/1051201658

[10] Sato, K. and Suzuki, A. (2011) Stochastic cash management problem with double exponential jump diffusion processes. Lecture Notes in Operations Research, 14, 186-194. 